Supporting Information for:

\title{
Flexible UV exposure sensor based on UV responsive polymer
}

Michele E. Lee and Andrea M. Armani*

Mork Family Department of Chemical Engineering and Materials Science, University of Southern California, Los Angeles, CA 90089, USA

*To whom correspondence should be addressed: armani@usc.edu

\section{Table of Contents}

1 Synthesis and Characterization of Photocleavable PMA …………..................................... 1

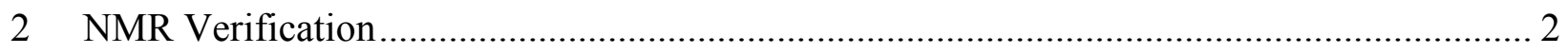

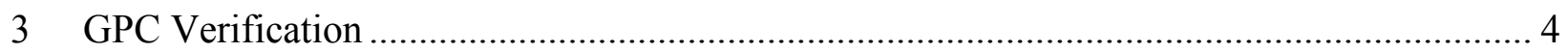

4 UV-Vis absorbance change in PMA with increased UV exposure ........................................ 5

5 Yellowness Index change of various concentrations of PMA film ........................................ 6

\section{Synthesis and Characterization of Photocleavable PMA}

Photocleavable polymer synthesis is outlined in Supplementary Scheme 1. Atom transfer radial polymerization reaction (ATRP) was carried out at $70{ }^{\circ} \mathrm{C}$ with a molar ratio $[\mathrm{M}]_{0}:[\mathrm{I}]_{0}:[\mathrm{PMDETA}]_{0}:[\mathrm{CuBr}]_{0}=400: 1: 2: 2,10 \%(\mathrm{v} / \mathrm{v})$ DMF. The reaction reached $35 \%$ conversion after $1 \mathrm{~h} 50 \mathrm{~min}$ and a final molecular weight of 13,000 Da, PDI 1.07. 


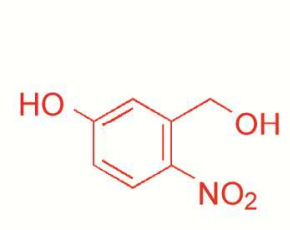

(1)

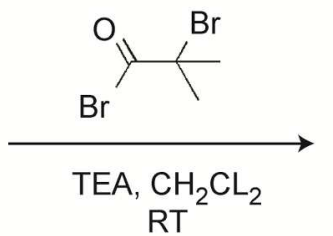

RT

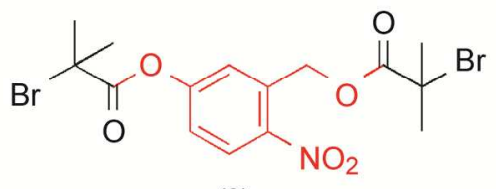

(2)

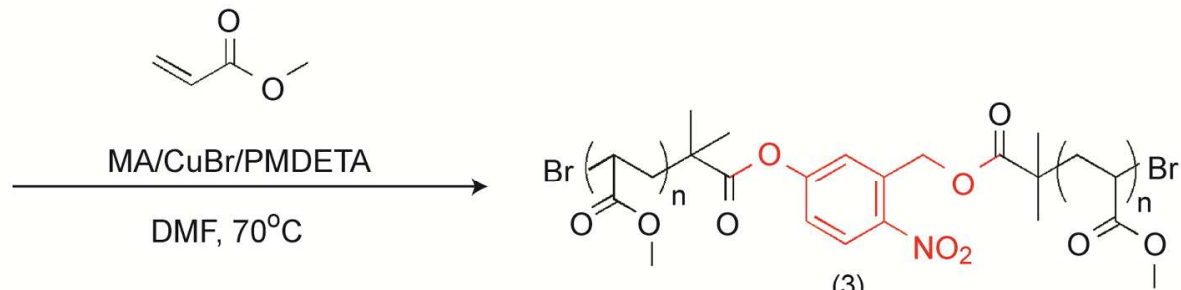

(3)

Supplementary Scheme 1. ATRP reaction outline. (1) is the ONB cleaving group, (2) is the bifunctional ATRP initiator, and (3) is the final UV responsive polymer.

\section{NMR Verification}

In order to confirm the polymer structure, ${ }^{1} \mathrm{H}$ signals were recorded on a $600 \mathrm{MHz}$ Varian spectrometer in THF- $\mathrm{d}_{8}$. Post-synthesis, signals corresponding to the PMA polymer were clearly observed between $1.5-2.5 \mathrm{ppm}$. In addition, signals between $7.0-8.5 \mathrm{ppm}$ corresponding to the pre-cleaved ONB group were as expected (Supplementary Fig. 1). The NMR sample was then subjected to $1 \mathrm{~h}$ UV exposure in a photoreactor. After UV exposure, a strong decrease in the ethyl group signal at 5.4ppm (peak d in Supplementary Fig. 1) indicates photocleavage of that bond (Supplementary Fig. 2). The broad spreading and decrease of aromatic peaks between 7.0 $8.5 \mathrm{ppm}$ indicates conversion of the original ONB group to other aromatic derivatives, i.e. the nitrosobenzaldehyde and azo dimer photoproducts.

Identification of all peaks: ${ }^{1} \mathrm{H}$ NMR $\left(600 \mathrm{MHz}, \mathrm{THF}-\mathrm{d}_{8}\right) \delta 10.81(1 \mathrm{H}, \mathrm{s}), 8.18(1 \mathrm{H}, \mathrm{d})$, $7.44(1 \mathrm{H}, \mathrm{s}), 7.37-7.35(1 \mathrm{H}, \mathrm{m}), 5.43(2 \mathrm{H}, \mathrm{d}), 4.37-4.31(2 \mathrm{H}, \mathrm{m}), 3.61(3 \mathrm{H}, \mathrm{s}), 2.41-2.28(1 \mathrm{H}, \mathrm{m})$, 1.93-1.85 (1H, m), 1.74-1.63 (1H, m), 1.60-1.44 (1H, m), 1.33-1.27 (6H, m), 1.21-1.15 (6H, m) 


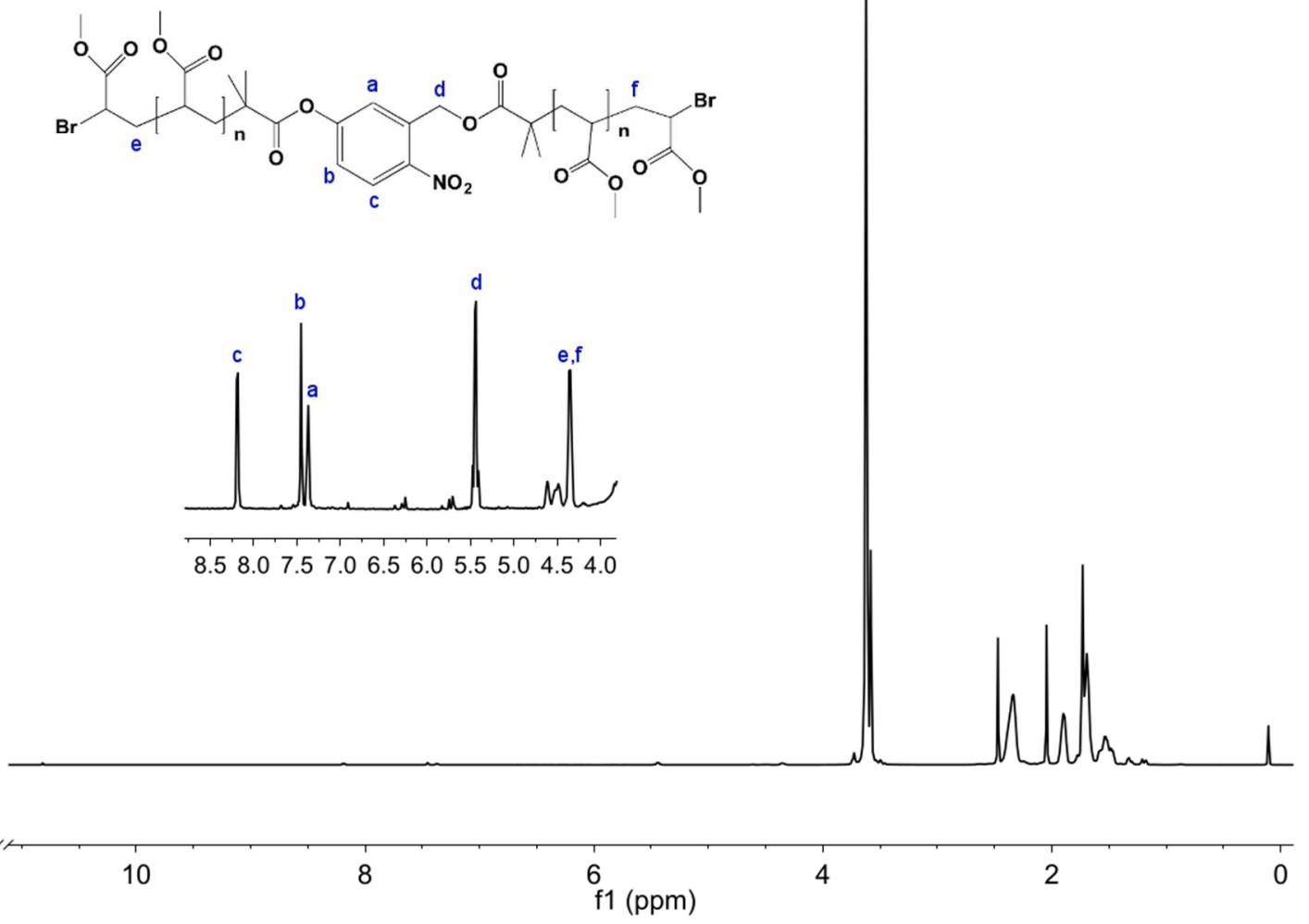

Supplementary Figure 1. ${ }^{1} \mathrm{H}$ NMR spectrum of ONB-modified PMA in THF- $\mathrm{d}_{8}$ before sun exposure. 

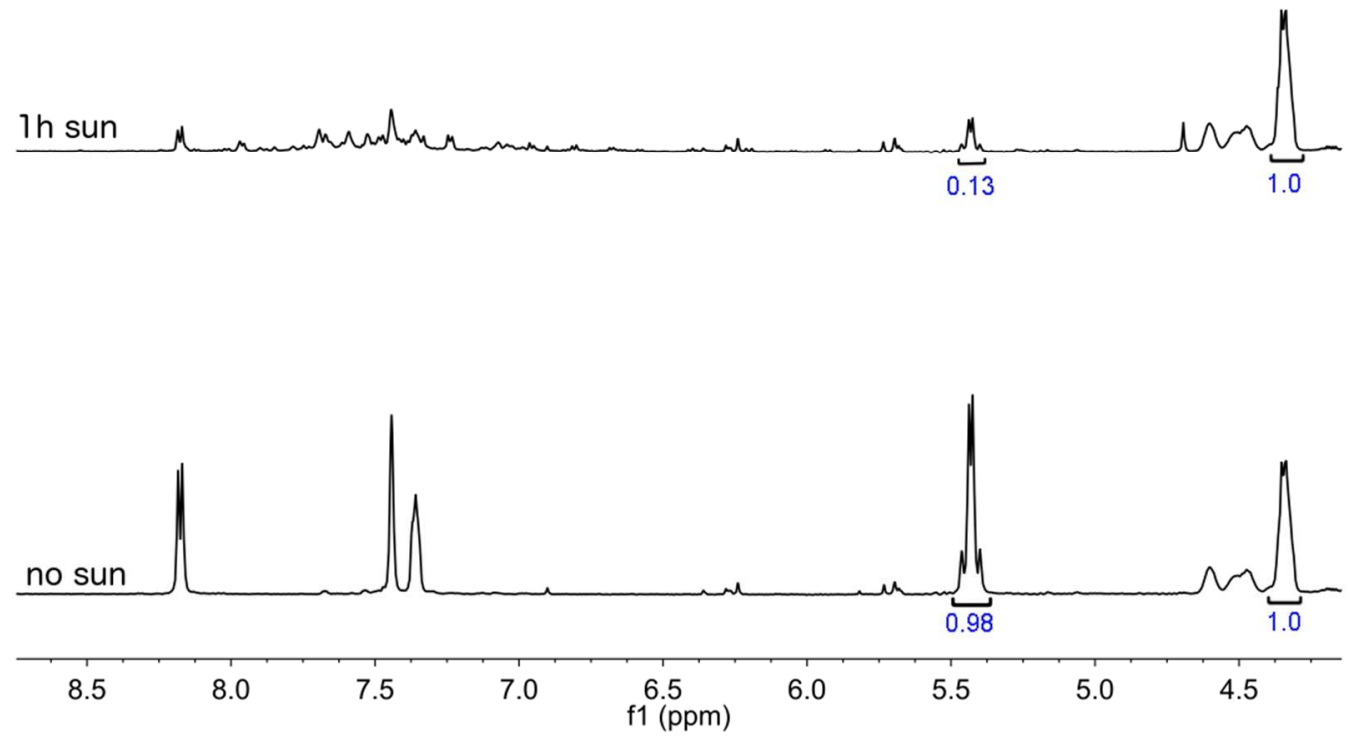

Supplementary Figure 2. ${ }^{1} \mathrm{H}$ NMR spectral comparison in THF-d 8 of ONB-modified PMA before (bottom) and after $1 \mathrm{~h}$ (top) simulated sun exposure.

\section{GPC Verification}

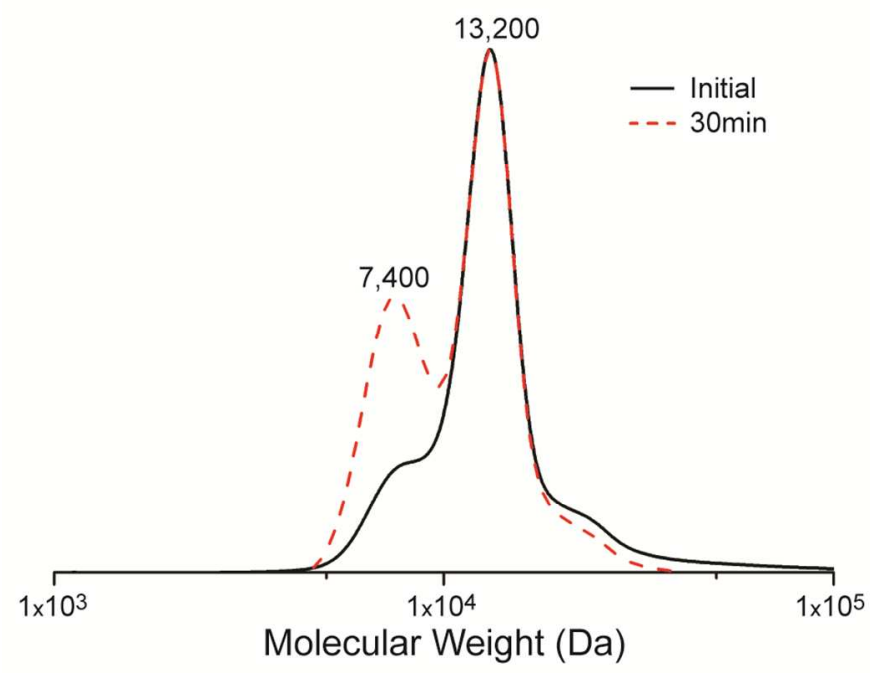

Supplementary Figure 3. GPC chromatogram showing reduction of PMA-ONB molecular weight by half after simulated sun exposure. 
In addition, photocleavage of the central ONB unit was characterized with GPC. A solid sample of PMA-ONB was exposed to simulated sunlight for 30 minutes, then dissolved in THF for GPC analysis. After exposure, there was a change in color of the sample from clear to light orange. GPC chromatograms of the polymer before and after exposure exhibit a clear shift of the PMA peak to approximately half of the original molecular weight (Supplementary Fig. 3), which confirms ONB placement in the center of the PMA chain.

\section{UV-Vis absorbance change in PMA with increased UV exposure}

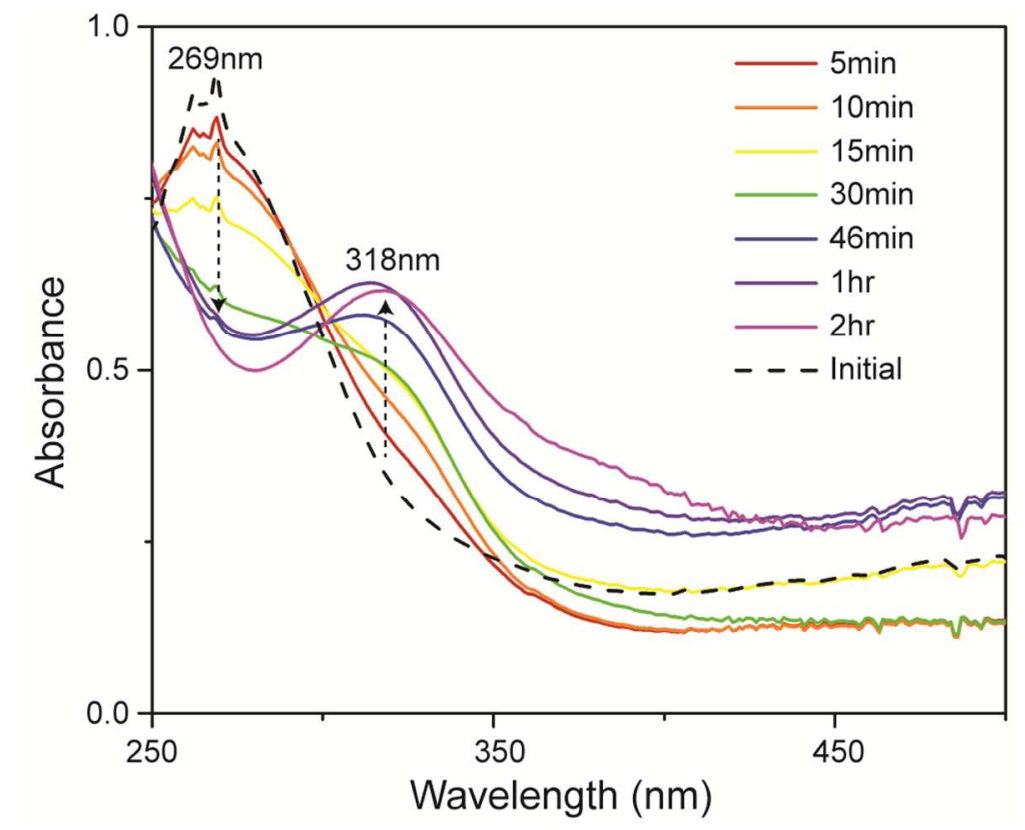

Supplementary Figure 4. Shift in sample absorbance peak with increasing simulated sun exposure time. 


\section{Yellowness Index change of various concentrations of PMA film}

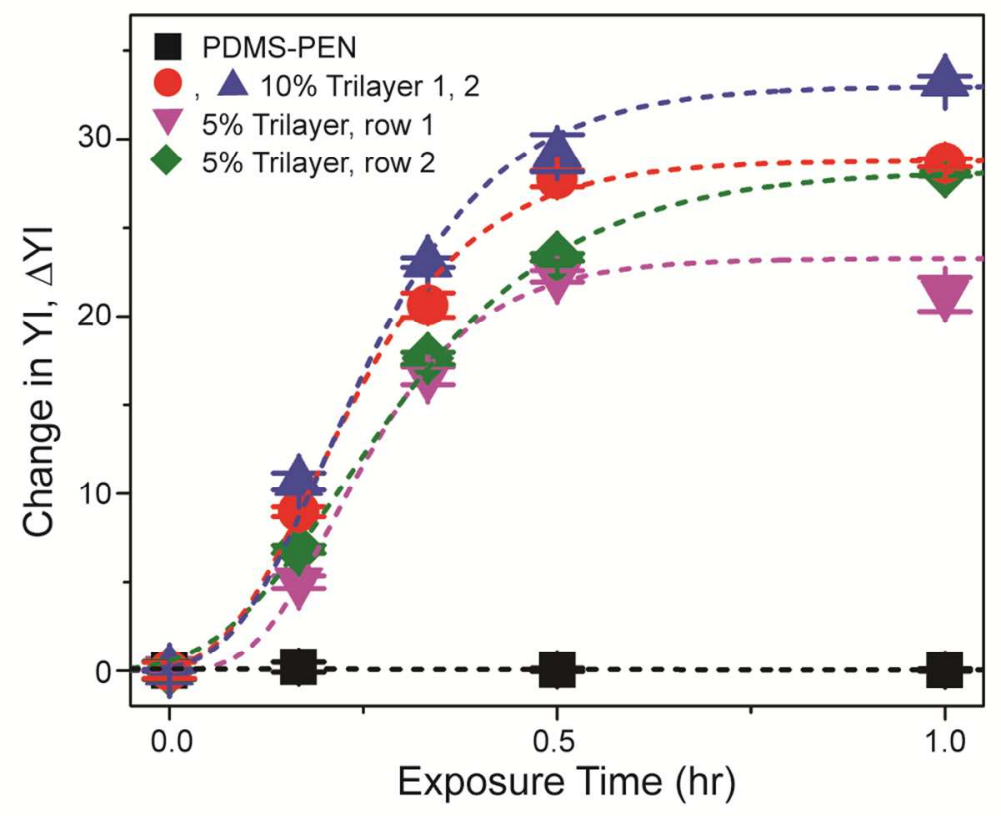

Supplementary Figure 5. Sigmoidal increase in yellowness index of trilayer sensors made from various concentrations of active PMA-ONB layer.

We tested multiple concentrations of PMA-ONB in ethyl acetate for drop coating to determine the optimal thickness to observe a significant color change after UV exposure. The $10 \mathrm{wt} \%$ concentration resulted in a stronger color change than the $5 \mathrm{wt} \%$ concentration due to increased layer thickness. However we did observe the same sigmoidal increase in $\Delta$ YI with exposure time in samples made from both concentrations (Supplementary Fig. 5), illustrating that the kinetic behavior of photocleavage is the same regardless of layer thickness. 